Pacific Journal of Mathematics

MENGER SPACES AND INVERSE LIMIT

DenNis J. Garity, James P. HeNDERson aNd David G. Wright 


\section{MENGER SPACES AND INVERSE LIMITS}

\section{Dennis J. Garity, James P. Henderson and David G. Wright}

M. Bestvina in 1984 characterized the Menger universal $n$-dimensional spaces. This characterization is used to identify certain inverse sequences having inverse limit homeomorphic to one of the Menger spaces. Specific models of the Menger spaces are then constructed in the Hilbert Cube as inverse limits of polyhedra. The union of these models is shown to be homeomorphic to the countably infinite dimensional space $\sigma$.

1. Introduction. In 1984, M. Bestvina [Be] characterized the Menger universal $n$-dimensional compactum $\mu_{n}$ as follows.

THEOREM. A space $X$ is homeomorphic to $\mu_{n}$ if and only if $X$ satisfies the following properties:

1. $X$ is compact and $n$-dimensional,

2. $X$ is $(n-1)$-connected $\left(C^{n-1}\right)$,

3. $X$ is locally $(n-1)$-connected $\left(L C^{n-1}\right)$, and

4. $X$ satisfies the disjoint $n$-cells property $\left(D D^{n} P\right)$.

Using this characterization, Bestvina showed that the various constructions in the literature of compact universal $n$-dimensional spaces ([Mg], $[\mathbf{L f}],[\mathbf{P a}])$ all yield $\mu_{n}$. In addition, Bestvina showed that each $\mu_{n}$ is homogeneous. Prior to this result, there had been characterizations only of $\mu_{0}$ (the Cantor set) and $\mu_{1}$ (the universal curve) [An].

Using Bestvina's characterization, we identify certain inverse sequences that have $\mu_{n}$ as inverse limit. This leads to the construction of models of $\mu_{n}$ in the Hilbert Cube. These models can be described by putting restrictions on the coordinates of points in the Hilbert Cube. We also show that the union of certain of these models naturally yields the countably infinite dimensional space $\sigma$.

2. Notation and terminology. All spaces are assumed to be separable and metrizable. A reference for dimension theory is [En]. A space is $n-1$ connected, $C^{n-1}$, if each map of $S^{k}, k \leq n-1$, into the space extends to a map of the $k+1$ cell into the space. A space $X$ is locally $n-1$ connected, $L C^{n-1}$, if for each point $p \in X$, and for each neighborhood $U$ of $p$ there is a neighborhood $V$ of $p, V \subset U$, so that each map of $S^{k}$ into $V, k \leq n-1$, extends to a map of $B^{k+1}$ into $U$. 
A space $X$ satisfies the disjoint $n$-cells property, $D D^{n} P$, if for each pair of maps $f, g: B^{n} \rightarrow X$ and for each $\varepsilon>0$ there are maps $f_{1}, g_{1}: B^{n} \rightarrow X$ so that $f_{1}\left(B^{n}\right) \cap g_{1}\left(B^{n}\right)=\varnothing$ and so that $d\left(f_{1}, f\right)<\varepsilon$, and $d\left(g_{1}, g\right)<\varepsilon$.

We denote an inverse sequence of topological spaces

$$
X_{0} \stackrel{p_{1}}{\leftarrow} X_{1} \stackrel{p_{2}}{\leftarrow} X_{2} \cdots
$$

by $\left\{X_{i}, p_{i}\right\}$. If $X$ is the inverse limit of such a sequence, we let $\pi_{i}: X \rightarrow X_{i}$ be projection onto the $i$ th coordinate space and let $p_{i j}: X_{j} \rightarrow X_{l}$, $j \geq i$, be the map induced by the bonding maps. If $\left\{X_{i}, p_{i}\right\}$ is an inverse sequence, we assume $X_{i}$ is metrized by a metric $d_{i}$ so that diameter $\left(X_{i}\right) \leq 1 / 2^{i}$. We use the metric $d(x, y)=\sum_{i=0}^{\infty} d_{i}\left(x_{l}, y_{l}\right)$ on the product space $\prod_{i=0}^{\infty} X_{i}$.

We view the Hilbert Cube $Q$ as $\prod_{i=1}^{\infty} I_{i}$ where $I_{i}=\left[0,1 / 2^{i}\right]$ and we view the $n$-cell $I^{n}$ as $\prod_{i=1}^{n} I_{i} \cdot Q_{j}$ is $\prod_{i=j}^{\infty} I_{i}$ so that for each $n, Q=I^{n} \times$ $Q_{n+1}$.

A number $x$ in $\left[0, \frac{1}{2}\right]$ that can be written as $k / 2^{n}, n \geq 1$, with $k$ and 2 relatively prime is called a dyadic rational of order $n$. So 0 and $\frac{1}{2}$ have order $1, \frac{1}{4}$ has order $2, \frac{1}{8}$ and $\frac{3}{8}$ have order 3 and so on.

3. Inverse sequences. There are a number of results in the literature giving conditions which imply that the inverse limit of $L C^{n}$ compacta is itself $L C^{n}$. Z. Cerin [Ce] shows that the inverse limit is $L C^{n}$ if and only if the inverse sequence is strongly $n e$-movable. L. McAuley and E. Robinson [M, R] show that the inverse limit is $L C^{n}$ if each bonding map is $U V^{n}$.

For the examples we are interested in, we need conditions that yield both $C^{n-1}$ and $L C^{n-1}$. Conditions 2 and 3 in the next Theorem are sufficient for this purpose.

TheOREM 1. Let $\left\{X_{i}, p_{i}\right\}$ be an inverse sequence of $L C^{n-1}$-dimensional compacta, satisfying the following conditions.

1. For each $i$ and map $f: B^{n} \rightarrow X_{i}$ there exists $j>i$ and maps $h_{1}, h_{2}$ : $B^{n} \rightarrow X_{j}$ with $h_{1}\left(B^{n}\right) \cap h_{2}\left(B^{n}\right)=\varnothing$ and $p_{i j} \circ h_{e}=$ f for $e=1,2$.

2. $X_{1}$ is $C^{n-1}$.

3. There is a constant $c$ so that for each $i$, for each map $f: B^{k+1} \rightarrow X_{i}$, $k \leq n-1$, and for each map $g: S^{k} \rightarrow X_{i+1}$ with $p_{i+1} \circ g=f \mid S^{k}$, there is an extension $h: B^{k+1} \rightarrow X_{i+1}$ with $p_{m, i+1} \circ h$ within $c / 2^{i+1}$ of $p_{m, i} \circ$ for each $m \leq i$.

Then $X=\lim _{\leftarrow}\left\{X_{i}, p_{i}\right\}$ is homeomorphic to $\mu_{n}$. 


\section{Proof.}

compact $n$-dimensional.

Since each $X_{i}$ is compact and $n$-dimensional, $X$ is compact and less than or equal to $n$-dimensional. We show below that $X$ is $L C^{n-1}$ and satisfies $D D^{n} P$. A standard argument similar to that in [Ca] then shows that maps from $B^{n}$ into $X$ are approximable by embeddings. In particular, $X$ contains $n$-dimensional subspaces and is thus $n$-dimensional.

\section{$D D^{n} P$.}

Let $f_{e}: B_{e}^{n} \rightarrow X, e=1,2$, be maps from $n$-cells $B_{1}^{n}, B_{2}^{n}$ into $X$ and let $\varepsilon>0$ be given. Choose $N$ so that diameter $\left[\prod_{i=N}^{\infty} X_{i}\right]<\varepsilon$. By conditions 2 and 3 , there is an arc $\alpha$ in $X_{N}$ connecting the image of $\pi_{N} \circ f_{1}$ to the image of $\pi_{N} \circ f_{2}$. We may view $\pi_{N} \circ f_{1}\left[B_{1}^{n}\right] \cup \alpha \cup \pi_{N} \circ f_{2}\left[B_{2}^{n}\right]$ as the image of the $n$-cell $B^{n}$ under a, map $g$. Furthermore, we may assume that $B_{e}^{n} \subset B^{n}$ with $\pi_{N} \circ f_{e}=g \mid B_{e}^{n}$. For a similar argument see [Ga].

Choose $j_{1}>N$ and maps $h_{1}, h_{2}: B^{n} \rightarrow X_{J_{1}}$ as in condition 1. Define $f_{e}^{1}: B_{e}^{n} \rightarrow X_{J_{1}}, e=1,2$, by $f_{e}^{1}=h_{e} \mid B_{e}^{n}$. Then

$$
f_{1}^{1}\left[B_{1}^{n}\right] \cap f_{2}^{1}\left[B_{2}^{n}\right]=\varnothing \quad \text { and } \quad \pi_{N} \circ f_{e}=P_{n, j_{1}} \circ f_{e}^{1} \text {. }
$$

Repeating this argument, we inductively define a sequence of integers $j_{1}<j_{2}<j_{3}<\cdots$ and maps $f_{e}^{m} \rightarrow X_{J_{m}}, e=1,2$, so that for each positive integer $m$,

$$
P_{j_{m}, J_{m+1}} \circ f_{e}^{m+1}=f_{e}^{m} .
$$

This procedure induces maps $g_{1}, g_{2}: B_{e}^{n} \rightarrow X$ with $\pi_{\iota} \circ g_{e}=\pi_{i} \circ f_{e}$ for $i \leq N$, and with $\pi_{j_{1}} \circ g_{1}\left[B_{1}^{n}\right] \cap \pi_{j_{1}} \circ g_{1}\left[B_{2}^{n}\right]=\varnothing$. It follows that $g_{e}$ is within $\varepsilon$ of $f_{e}$ and that $g_{1}\left[B_{1}^{n}\right] \cap g_{2}\left[B_{2}^{n}\right]=\varnothing$.

$C^{n-1}$.

Let $\varepsilon_{\imath}=c / 2^{\imath}$. Let $f: S^{k} \rightarrow X, k \leq n-1$, be given. Since $X_{1}$ is $C^{n-1}$, $\pi_{1} \circ f$ extends to a map $f_{1}: B^{k+1} \rightarrow X_{1}$. Use condition 3 to extend $\pi_{2} \circ f$ to a map $f_{2}: B^{k+1} \rightarrow X_{2}$ so that

$$
d\left(f_{1}, p_{2} \circ f_{2}\right)<\varepsilon_{2} .
$$

In this manner we inductively define a sequence of maps $f_{m}: B^{k+1} \rightarrow$ $X_{m}$ with

$$
d\left(p_{i, m} \circ f_{m}, p_{t, m-1} \circ f_{m-1}\right)<\varepsilon_{m} \quad \text { for } i \leq m-1,
$$

and so that $f_{m} \mid S^{k}=\pi_{m} \circ f$. 
Define a map $g: B^{k+1} \rightarrow \prod_{i=1}^{\infty} X_{i}$ by

$$
g_{i}(x)=\lim _{m \rightarrow \infty} p_{i_{m}} \circ f_{m}(x) \text {. }
$$

Since the sequence $\left(p_{i_{m}} \circ f_{m}\right)_{m=1}^{\infty}$ is uniformly Cauchy, each $g_{i}$ is continuous. The definition of the functions $f_{m}$ shows that $g_{i}$ extends $\pi_{i} \circ f$. Finally, since $p_{i-1, i} \circ g_{i}=g_{i-1}$,

$$
g\left(B^{k+1}\right) \subset X .
$$

Then the required extension has been constructed.

$L C^{n-1}$.

Given $q \in X$ and $\varepsilon>0$, choose $N$ so that diameter $\left(\prod_{i=N}^{\infty} X_{i}\right)<\varepsilon / 2$ and so that

$$
\sum_{i=N}^{\infty} \varepsilon_{i}<\frac{\varepsilon}{8 N}
$$

Use the fact that $X_{N}$ is $L C^{n-1}$ to choose $\delta_{1}<\delta_{2}<\varepsilon / 8 N$ so that any map of $S^{k}, k \leq n-1$, into the $\delta_{1}$ neighborhood of $q_{N}$ in $X_{N}$ extends to a map of $B^{k+1}$ into the $\delta_{2}$ neighborhood of $q_{N}$ in $X_{N}$. We may also require that any set of diameter $<2 \delta_{2}$ in $X_{N}$ has image in $X_{i}$ of diameter $<\varepsilon / 8 N$ under the map $p_{i N}$. This uses the uniform continuity of the bonding maps.

Let $f: S^{k} \rightarrow X$ be a map into the $\delta_{1}$ neighborhood of $q$ in $X$. Then $\pi_{N} \circ f\left(S^{k}\right)$ is contained in the $\delta_{1}$ neighborhood of $q_{N}$, and so there is an extension $g_{N}: B^{k+1} \rightarrow X_{N}$ with image of diameter $<\delta_{2}$.

For each $i \leq N$, we thus have maps $g_{i} \equiv p_{i, N} \circ g_{N}: B^{k+1} \rightarrow X_{i}$ with image of diameter $<\varepsilon / 8 N$, and with $g_{i} \mid S^{k}=\pi_{i} \circ f$.

Since $\sum_{i=N}^{\infty} \varepsilon_{i}<\varepsilon / 8 N$, we may now proceed exactly as in the proof of $C^{n-1}$ to construct an extension $h: B^{k+1} \rightarrow X$ of $f$ so that

$$
d\left(\pi_{i} \circ h, g_{i}\right)<\frac{\varepsilon}{8 N} \quad \text { for } i \leq N \text {. }
$$

It follows that $h_{t}$ is a map into the $4 \cdot \varepsilon / 8 N=\varepsilon / 2 N$ neighborhood of $q_{i}$ in $X_{1}$ for $i \leq N$.

Because $\operatorname{diam}\left(\prod_{i=N}^{\infty} X_{i}\right)<\varepsilon / 2$, it then follows that $h$ is a map into the $\varepsilon$ neighborhood of $q$ in $X$.

\section{Specific models in the Hilbert cube.}

THeOREM 2. Fix $n \geq 0$. Let $P_{i} \subset I^{i}, i \geq n$, be a sequence of compact n-dimensional LC $\mathrm{LC}^{n-1}$ spaces so that

(a) $P_{i} \times\left\{0,1 / 2^{i+1}\right\} \subset P_{i+1}$ and $P_{i+1} \subset P_{i} \times I_{i+1}$,

(b) $P_{n}$ is $C^{n-1}$, and

(c) There is a constant $c$ so that for each map $f: B^{k+1} \rightarrow P_{i} \times I_{i+1}$ 
( $k \leq n-1)$ with $f\left(S^{k}\right) \subset P_{i+1}$, there is a map $g: B^{k+1} \rightarrow P_{l+1}$ with $d(f, g)<c / 2^{i+1}$ and with $f\left|S^{k}=g\right| S^{k}$.

Let $X=\bigcap_{l=n}^{\infty}\left(P_{i} \times Q_{l+1}\right)$. Then $X \cong \mu_{n}$.

Proof. $X$ is homeomorphic to $\lim _{\leftarrow}\left\{P_{i}, p_{i}\right\}$ where $p_{i}$ is the restriction of projection from $I^{i}$ onto $I^{i-1}$. So it suffices to check that conditions 1 , 2 , and 3 from Theorem 1 are satisfied.

Conditions (a) and (b) abnve directly imply that conditions 1 and 2 are satisfied.

For condition 3, let $f: B^{k+1} \rightarrow P_{i}, k \leq n-1$, and $g: S^{k} \rightarrow P_{i+1}$ be maps with

$$
p_{i+1} \circ g=f \mid S^{k} \text {. }
$$

Extend $g$ to a map $h: B^{k+1} \rightarrow P_{i} \times I_{i+1}$ so that $p_{i+1} \circ h=f$. Use condition (c) to approximate $h$ by a map

$$
\begin{gathered}
h_{1}: B^{k+1} \rightarrow P_{i+1}, \quad \text { with } \\
d\left(h, h_{1}\right)<\frac{c}{2^{i+1}}, \quad \text { and with } h_{1}\left|S^{k}=h\right| S^{k} .
\end{gathered}
$$

Then $p_{J, l+1} \circ h_{1}$ is within $c / 2^{i+1}$ of $p_{j i} \circ f$ for each $j \leq i$ and condition 1 is satisfied.

We now construct a specific model satisfying the conditions in Theorem 2. Again, fix $n \geq 0$.

For $X=I^{i}$ or $Q$, let

$$
X_{*} \equiv\left\{x \in X \mid \text { for each choice of } n+1 \text { coordinates } x_{m_{1}}, \ldots, x_{m_{n+1}}\right. \text { of }
$$

$$
X \text { with } m_{1}<\cdots<m_{n+1} \text {, at least one of the }
$$

$$
\text { coordinates is dyadic of order } \left.\leq m_{n+1}\right\} \text {. }
$$

Let $P_{l}=I_{*}^{l}$.

For $n=1$, the one-dimensional polyhedra $P_{1}, P_{2}$, and $P_{3}$ are illustrated in Figure 1.

When $n=0, P_{i}=\prod_{j=1}^{i}\left\{0,1 / 2^{j}\right\}$, the corner points of the $i$-cell $I^{i}$.

$$
P_{1}
$$

$P_{2}$

$P_{3}$
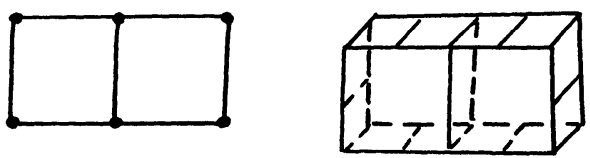
Let $X_{n}=\bigcap_{i=n}^{\infty}\left(P_{l} \times Q_{l+1}\right)$. Then $X_{n}$ is $Q_{*}$. Note that $X_{0}$ is the Cantor Set consisting of the corner points of the Hilbert Cube. In Theorem 3 below, we show that $X_{n} \cong \mu_{n}$. Before proving this theorem, we provide an alternate description of $P_{l}$ that is easier to work with.

Fix $n$. Let $P_{n}^{\prime}=I^{n}$. Let $P_{n}^{\prime}$ be viewed as a cell complex consisting of rectilinear $n$-cells with sides of length $1 / 2^{n+1}$ by subdividing each factor $I_{i}$ of $I^{n}$ into subintervals of lengths $1 / 2^{n+1}$. Let $A_{n}$ be the $(n-1)$ skeleton of this cell complex.

Define $P_{n+1}^{\prime} \subset I^{n+1}$ as

$$
A_{n} \times I_{n+1} \cup P_{n}^{\prime} \times\left\{0, \frac{1}{2^{n+1}}\right\} .
$$

Note that $P_{n+1}^{\prime}$ can be viewed as a cell complex consisting of rectilinear $n$-cells with sides of length $1 / 2^{n+2}$ by subdividing each factor $I_{i}$ of $I^{n+1}$ into subintervals of length $1 / 2^{n+2}$.

Inductively assume $P_{j}^{\prime} \subset I^{\prime}$ has been defined so that $P_{J}^{\prime}$ can be viewed as a cell complex consisting of rectilinear $n$-cells with sides of length $1 / 2^{j+1}$ by subdividing each factor $I_{i}$ of $I^{j}$ into subintervals of length $1 / 2^{j+1}$.

Let $A_{j}$ be the $n-1$ skeleton of this cell complex. Define $P_{j+1}^{\prime} \subset I^{j+1}$ as

$$
A_{j} \times I_{j+1} \cup P_{j}^{\prime} \times\left\{0,1 / 2^{j+1}\right\}
$$

LeMma 1. For each $i, P_{i}=P_{i}^{\prime}$.

Proof. The proof proceeds by induction. When $i=n, P_{l}=P_{i}^{\prime}=I^{n}$.

Assume inductively that $P_{i}=P_{i}^{\prime}$. Let $x$ be a point in $P_{i+1}$. We may view $x$ as $(a, b)$ where $a=\left(x_{1}, \ldots, x_{i}\right)$ and $b=x_{i+1}$. Then $a \in P_{i}=P_{i}^{\prime}$. If $i-n+1$ coordinates of $a$ have order $\leq i+1$, then $a \in A_{i}$ and so $(a, b) \in P_{i+1}^{\prime}$. If fewer than $i-n+1$ coordinates of $a$ have order $\leq i+$ 1 , then $b$ must have order $i+1$. So $b \in\left\{0,1 / 2^{i+1}\right\}$ and $(a, b) \in P_{i+1}^{\prime}$.

Conversely, let $X=(a, b) \in P_{i+1}^{\prime}$. Then $a \in P_{i}^{\prime}=P_{i}$. If $b \in$ $\left\{0,1 / 2^{i+1}\right\}$, then $(a, b) \in P_{i+1}$. If $b \notin\left\{0,1 / 2^{i+1}\right\}$, then $a$ must have $i-n+1$ coordinates of order $\leq i+1$ since $a$ is then in $A_{i}$. Again $(a, b) \in P_{i+1}$.

THEOREM 3. $X_{n} \cong \mu_{n}$.

Proof. The alternate description of the $P_{i}$ given by Lemma 1 shows that each $P_{i}$ is a compact $n$-dimensional polyhedron. Thus each $P_{i}$ is $L C^{n-1}$. So it suffices to show that the $P_{l} \subset I^{l}$ satisfy conditions (a), (b), 
and (c) in Theorem 2. The alternate description of the $P_{i}$ shows directly that condition $a$ is satisfied.

$P_{n}$ is an $n$-cube and hence is $(n-1)$-connected. Therefore condition (b) is satisfied. For condition (c), let $f$ be a map from $B^{k+1}$ to $P_{i} \times I_{i+1}$ $(k \leq n-1)$ with $f\left(S^{k}\right) \subset P_{i+1}$. Using the alternate description, $P_{i+1}$ may be viewed as the $n$-skeleton of a cell complex $L$ with underlying space $P_{\imath} \times I_{1+1}$. We may assume that $L$ consists entirely of rectilinear $(n+1)$ cells with sides of length $1 / 2^{i+1}$.

Let $\sigma$ be such an $n+1$ cell of $L$. Since $k+1 \leq n, f \mid f^{-1}(\sigma)$ : $f^{-1}(\sigma) \rightarrow \sigma$ may be replaced by a map $g: f^{-1}(\sigma) \rightarrow \partial \sigma$ so that $g \mid f^{-1}(\partial \sigma)$ $=f \mid f^{-1}(\partial \sigma)[\mathbf{H}, \mathbf{W}]$. It follows that $d\left(f\left|f^{-1}(\sigma), g\right| f^{-1}(\boldsymbol{\sigma})\right) \leq$ $\operatorname{diameter}(\sigma)=(n+1) / 2^{i+1}$.

By following the above procedure on each $n+1$ cell of $L$, one obtains a map $g: B^{k+1} \rightarrow P_{i+1}$ so that $g\left|S^{k}=f\right| S^{k}$, and so that $d(f, g)$ $<(n+1) / 2^{i+1}$.

5. Menger spaces and $\sigma$. In this section, we show that if $X=$ $\cup_{n=1}^{\infty} X_{n}$, then $X$ is homeomorphic to $\sigma$. Recall that $\sigma$ may be viewed as the set of points in Hilbert space having at most finitely many nonzero coordinates. In order to obtain the desired goal, we will show that $X$ satisfies the following characterization $[\mathbf{H e}]: X$ is a $\sigma$-manifold if and only if:

1. $X$ is an ANR.

2. $X$ is the countable union of finite dimensional compacta.

3. Each compact subset of $X$ is a strong $Z$-set in $X$.

4. For each integer $k$, mapping $f: \mathbf{R}^{k} \rightarrow X$, and $\varepsilon: X \rightarrow(0,1)$, there is an injection $f^{\prime}: \mathbf{R}^{k} \rightarrow X$ with $d\left(f(x), f^{\prime}(x)\right)<\varepsilon(x)$.

The last property is referred to as the Euclidean injection property (EIP). Condition 3 means that if $A$ is a compact subset of $X$, for each open cover $\mathscr{U}$ of $X$ and sequence of mappings $\alpha_{1}, \alpha_{2}, \ldots$ of $Q$ into $X$, there are $\mathscr{U}$-approximations $\beta_{1}, \beta_{2}, \ldots$ such that $\bigcup\left\{\beta_{i}(Q): 1 \leq i<\infty\right\}$ misses a neighborhood of $A[\mathbf{B}, \mathbf{B}, \mathbf{M}, \mathbf{W}]$. Condition 2 is satisfied since each $X_{n}$ is a compact finite dimensional set. The space $X$ will be shown to satisfy the other conditions through a sequence of results.

The first lemma involves approximating mappings of $\mathbf{R}^{k}$ into $Q$ by mappings into $X$. Throughout the remainder, by a basic open set $V$ in $Q$ we will mean an open set in $Q$ of the form $V=\left(\Pi_{i=1}^{n} V_{i}\right) \times Q_{n+1}$ where $V_{i}$ is a connected open set in $I_{i}$. We let $D_{k}=\left\{\left(x_{i}\right) \in Q: x_{i}=0\right.$ for $\left.i>k\right\}$ and set $D=\bigcup_{n=0}^{\infty} D_{k}$.

LEMMA 2. Let $V$ be a basic open set of $Q$. For $f: \mathbf{R}^{k} \rightarrow V$ and $\varepsilon$ : $\mathbf{R}^{k} \rightarrow(0,1)$, there is a mapping $f^{\prime}: \mathbf{R}^{k} \rightarrow D$ with $d\left(f(x), f^{\prime}(x)\right)<\varepsilon(x)$. 
Proof. Let $f_{i}$ be the $i$ th coordinate function of $f$. Define the $i$ th coordinate function $f_{i}^{\prime}$ of a function $f^{\prime}: \mathbf{R}^{k} \rightarrow V$ by

$$
f_{i}^{\prime}(x)= \begin{cases}f_{i}(x) & \text { if } i \leq n \\ \operatorname{Max}\left\{f_{i}(x)-\frac{\varepsilon(x)}{2^{i}}, 0\right\} & \text { if } i>n\end{cases}
$$

Clearly, $d\left(f(x), f^{\prime}(x)\right)<\varepsilon(x)$.

We now turn to the problem of showing that $X$ is an ANR. According to Dugundji [Du], it suffices to show that given any open cover $\mathscr{U}$ of $X$, there is an open cover $\mathscr{V}$ of $X$ such that given any simplicial complex $K$, any partial realization of $K$ in $\mathscr{V}$ extends to a full realization of $K$ in $\mathscr{U}$. A partial realization of $K$ in $\mathscr{U}$ is a mapping $h: L \rightarrow X$ in which $L$ is a subcomplex of $K$ containing every vertex of $K$ and such that the sets $h(|L \cap s|)$ refine $\mathscr{U}$ where $s$ is a simplex of $K$. A full realization of $K$ in $\mathscr{U}$ is a partial realization of $K$ in $\mathscr{U}$ where $K=L$.

Proposition 1. $X$ is an $A N R$.

Proof. Let $\mathscr{U}$ be an open cover of $X$. Choose $\mathscr{V}$ to be a locally finite open cover of $X$ refining $\mathscr{U}$ such that each open set in $\mathscr{V}$ is of the form $V^{\prime}=V \cap X$ where $V$ is a basic open set in $Q$. Given a partial realization $h: L \rightarrow X$ in $\mathscr{V}$, it suffices to show that a mapping $h: \operatorname{Bd} I^{k} \rightarrow V^{\prime} \in \mathscr{V}$ can be extended to $h^{\prime}: I^{k} \rightarrow V^{\prime}$ to conclude that $h$ can be extended to a full realization of $K$ in $\mathscr{V}$, and hence in $\mathscr{U}$. Given a $k$-simplex $s$ in $K$, the image of all vertices of $s$ lie in at least one element $V^{\prime}$ of $\mathscr{V}$. If $V_{s}^{\prime}=\bigcap\left\{V^{\prime} \in \mathscr{V}:\right.$ the image of all vertices of $s$ lie in $\left.V^{\prime}\right\}$, then $V_{s}^{\prime}=V_{s} \cap$ $X$ where $V_{s}$ is a basic open set. Thus extending the mapping of $|L \cap s|$ to $|s|$ in $V_{s}$ extends the mapping in each basic set $V^{\prime} \in \mathscr{V}$ containing the image of the vertices of $s$. Since $V_{s}$ is contractible, $h: \mathrm{Bd} I^{k} \rightarrow V_{s}^{\prime}$ can be extended to $h_{1}: I^{k} \rightarrow V_{s}$. It now follows from Lemma 2 that the mapping $h_{1}$ restricted to the interior of $I^{k}$ can be approximated by a mapping $g$ : int $I^{k} \rightarrow V_{s}^{\prime}$ which extends by $h$ to all of $I^{k}$, yielding $h^{\prime}: I^{k} \rightarrow V_{s}^{\prime}$.

Proposition 2. X satisfies the EIP.

Proof. Recall that $D=\left\{\left(x_{i}\right) \in Q: x_{i}=0\right.$ for almost all $\left.i\right\}$. By Lemma 2, it suffices to show that $D$ has the EIP. Let $f: R^{k} \rightarrow D$ and $\varepsilon$ : $R^{k} \rightarrow(0,1)$ be given. Let $\Pi: Q \rightarrow I^{2 k+1}$ be projection onto the first $2 k+1$ coordinates. It is well-known $[\mathbf{H}, \mathbf{W}]$ that a map of $R^{k}$ into $I^{2 k+1}$ 
can be approximated by an injection. Let $g: R^{k} \rightarrow I^{2 k+1}$ be such that $d(\Pi \circ f(x), g(x))<\varepsilon(x)$ for each $x$ in $R^{k}$. Define $f^{\prime}: R^{k} \rightarrow D$ to be a map whose first $2 k+1$ coordinates are given by $g$ and whose remaining coordinates are the same as $f$. Then $f^{\prime}$ shows that $D$ has the EIP.

The final necessary result is that each compact subset of $X$ be a strong $Z$-set. This will be accomplished by first showing that each compact subset of $X$ is a $Z$-set in $Q$, and then getting the stronger property in $X$. Recall that a closed subset $A$ of an ANR $X$ is a $Z$-set if the relative homology groups $H_{*}(U, U-A ; Z)=0$ for each open set $U$ in $X$ and $A$ is 1-LCC embedded in $X$.

Proposition 3. $X_{n}$ is a Z-set in $Q$.

Proof. Since $X_{n}$ is a finite dimensional subset of $Q$,

$$
H_{*}\left(U, U-X_{n} ; Z\right)=0
$$

$[\mathbf{D}, \mathbf{W}]$. Thus it suffices to show that $X_{n}$ is 1-LCC in $Q$. Let $p$ be a point in $X_{n}$ and $U$ be an open set containing $p$. Choose $V$ to be a basic open set containing $p$ with $V \subset U$. For $g: S^{1} \rightarrow V-X_{n}$, there is a homotopy $h_{t}: S^{1} \rightarrow V$ with $h\left(S^{1} \times I\right) \subset V-X^{n}, h_{0}=g$, and $h_{1}\left(S^{1}\right) \subset D_{k} \cap V$ for some $k>n+2$. Since $X_{n} \cap D_{k}$ is a tame, $n$-dimensional subpolyhedron of $D_{k}, h_{1}\left(S^{1}\right)$ contracts in $D_{k} \cap V$ missing $X_{n}$, and $X_{n}$ is $1-\mathrm{LCC}$ in $Q$.

Corollary. Each compact subset $C$ of $X$ is a Z-set in $Q$.

Proof. Since $C=\cup_{n=1}^{\infty}\left(X_{n} \cap C\right)$, and $X_{n} \cap C$ is a $Z$-set in $Q$, it follows $[\mathbf{C}, \mathbf{D}, \mathbf{M}]$ that $C$ is a $Z$-set in $Q$.

LeMma 3. For each open cover $\mathscr{V}$ of $Q$ and each map $f: Q \rightarrow Q$ there is a $\mathscr{V}$-approximation $f^{\prime}$ so that $f^{\prime}(Q) \subset D$.

Proof. The proof is similar to the proof of Lemma 2 and is left to the reader.

Proposition 4. Every compact subset of $X$ is a strong Z-set in $X$.

Proof. Let $C$ be a compact subset of $X, \mathscr{U}$ an open cover of $X$, and $\left\{\alpha_{i}\right\}$ a sequence of mappings of $Q$ into $X$. We may suppose that $\mathscr{U}$ is the restriction of an open cover $\mathscr{W}$ of $Q$. Let $\mathscr{V}$ be an open star refinement of $\mathscr{W}$. Since $C$ is a $Z$-set in $Q$ and hence a strong $Z$-set in $Q[\mathbf{B}, \mathbf{B}, \mathbf{M}, \mathbf{W}]$, 
there are $\mathscr{V}$-approximations $\beta_{1}^{\prime}$ to $\alpha_{i}$ in $Q$ such that for some neighborhood $N$ of $C$ in $Q, \beta_{i}^{\prime}(Q)$ misses $N$ for each $i$. Let $M$ be a neighborhood of $C$ in $Q$ whose closure is contained in $N$. By Lemma 3, each $\beta_{i}^{\prime}$ has a $\mathscr{V}$-approximation $\beta_{i}$ that takes $Q$ into $D$. We may further assume that each approximation $\beta_{i}$ is so close to $\beta_{i}^{\prime}(Q)$ misses $M$. Since $\beta_{i}^{\prime}$ is a $\mathscr{V}$-approximation of $\alpha_{i}$ and $\beta_{i}$ is a $\mathscr{V}$-approximation of $\beta_{i}^{\prime}, \beta_{i}$ is a $\mathscr{W}$-approximation of $\alpha_{i}$. However, $\beta_{i}(Q)$ is contained in $D$ which lies in $X$, so $\beta_{i}$ is a $\mathscr{U}$-approximation of $\alpha_{i}$, and our proof is complete.

\section{THEOREM 4. $X$ is homeomorphic to $\sigma$.}

Proof. Since $X$ satisfies the characterization theorem, $X$ is a $\sigma$-manifold. We have not shown that $X$ is homeomorphic to $\sigma$. However, a $\sigma$-manifold may be factored as $|K| \times \sigma$ where $K$ is a countable, locally finite simplicial complex [Ch]. It follows from Lemma 2 that $\pi_{n}(X)=0$ for all $n$, so $\pi_{n}(|K|)=0$ for all $n$, and $|K|$ is contractible. Thus $X$ is contractible and homeomorphic to $\sigma$ since they have the same homotopy type [Ch].

It should be noted that a more general result follows from the above proofs. The following theorem is immediate.

THEOREM 5. Let $X=\bigcup_{n=1}^{\infty} X_{n}$, where each $X_{n}$ is a compact, finite dimensional $Z$-set in $Q$, with $X$ containing the set of all points in $Q$ having at most finitely many nonzero coordinates. Then $X$ is homeomorphic to $\sigma$.

Note that this immediately implies that $D$ is homeomorphic to $\sigma$.

\section{REFERENCES}

[An] R. D. Anderson, A characterization of the universal curve and a proof of its homogeneity, Ann. of Math., 67 (1958), 313-324.

[Be] M. Bestvina, Characterizing k-dimensional universal Menger compacta, Ph.D. Dissertation, 1984, University of Tennessee, Knoxville.

[B,B,M,W] M. Bestvina, P. Bowers, J. Mogilski, and J. Walsh, Characterization of Hilbert space manifolds revisited, preprint.

[Ca] J. W. Cannon, Shrinking cell-like decompositions of manifolds. Codimension three, Annals of Math., 110 (1979), 83-112.

[Ce] Z. Cerin, Characterizing global properties in inverse limits, Pacific J. Math., 112 (1984), 49-68.

[Ch] T. A. Chapman, Dense sigma-compact subsets of infinite-dimensional manifolds, Trans. Amer. Math. Soc., 154 (1971), 399-426.

[C,D,M] D. Curtis, T. Dobrowolski, and J. Mogilski, Some applicaqtions of the topological characterizations of the sigma-compact spaces $l_{f}^{2}$ and $\Sigma$, preprint. 
[D,W] R. J. Daverman and J. J. Walsh, Cech homology characterizations of infinite dimensional manifolds, Amer. J. Math., 103 (1981), 411-435.

[Du] J. Dugundji, Absolute neighborhood retracts and local connectedness in arbitrary metric spaces, Compositio Math., 13 (1958), 229-246.

[En] R. Engelking, Dimension Theory, North Holland, New York, 1978.

[Ga] D. Garity, $A$ characterization of manifold decompositions satisfying the disjoint triples property, Proc. Amer. Math. Soc., 83 (1981), 833-838.

[He] J. P. Henderson, Recognizing o-manifolds, Proc. Amer. Math. Soc., 94 (1985), 721-727.

[H,W] W. Hurewicz and H. Wallman, Dimension Theory, Princeton University Press, Princeton, 1974.

[Lf] S. Lefschetz, On compact spaces, Ann. of Math., 32 (1931), 521-538.

$[\mathrm{M}, \mathrm{R}] \quad$ L. F. McAuley and E. E. Robinson, On inverse convergence of sets, inverse limits and homotopy regularity, Houston J. Math., 8 (1982), 369-388.

[Mg] K. Menger, Kurventheorie, Teubner, Berlin-Leipzig, 1932.

[Pa] B. A. Pasynkov, Partial topological products, Trans. Moscow Math., Soc., 13 (1965), 153-271.

[Wa] J. J. Walsh, A class of spaces with infinite cohomological dimension, Michigan Math. J., 27 (1980), 215-222.

Received September 29, 1986.

Oregon State University

CORVALLIS, OR 97331

The Colorado College

Colorado Springs, CO 80903

AND

BRIGHAM YOUNG UNIVERSITY

PROVO, UT 84602 



\section{PACIFIC JOURNAL OF MATHEMATICS EDITORS}

\author{
V. S. VARADARAJAN \\ (Managing Editor) \\ University of California \\ Los Angeles, CA 90024 \\ HERBERT ClEMENS \\ University of Utah \\ Salt Lake City, UT 84112 \\ R. FINN \\ Stanford University \\ Stanford, CA 94305
}

\author{
HERMANN FLASCHKA \\ University of Arizona \\ Tucson, AZ 85721
}

RAMESh A. GANGolli

University of Washington Seattle, WA 98195

VAUGHAN F. R. JONES

University of California

Berkeley, CA 94720

\author{
ROBION KIRBY \\ University of California \\ Berkeley, CA 94720
}

C. C. MOORE

University of California

Berkeley, CA 94720

HAROLD STARK

University of California, San Diego

La Jolla, CA 92093

\section{ASSOCIATE EDITORS}
R. AREnS
E. F. BECKENBACH
B. H. NEUMANN
F. WOLF
K. YOSHIDA

(1906-1982)

\section{SUPPORTING INSTITUTIONS}

\begin{abstract}
UNIVERSITY OF ARIZONA
UNIVERSITY OF BRITISH COLUMBIA

CALIFORNIA INSTITUTE OF TECHNOLOGY

UNIVERSITY OF CALIFORNIA

MONTANA STATE UNIVERSITY

UNIVERSITY OF NEVADA, RENO

NEW MEXICO STATE UNIVERSITY

OREGON STATE UNIVERSITY
\end{abstract}

\author{
UNIVERSITY OF OREGON \\ UNIVERSITY OF SOUTHERN CALIFORNIA \\ STANFORD UNIVERSITY \\ UNIVERSITY OF HAWAII \\ UNIVERSITY OF TOKYO \\ UNIVERSITY OF UTAH \\ WASHINGTON STATE UNIVERSITY \\ UNIVERSITY OF WASHINGTON
}

The Supporting Institutions listed above contribute to the cost of publication of this Journal, but they are not owners or publishers and have no responsibility for its content or policies.

Mathematical papers intended for publication in the Pacific Journal of Mathematics should be in typed form or offset-reproduced (not dittoed), double spaced with large margins. Please do not use built up fractions in the text of the manuscript. However, you may use them in the displayed equations. Underline Greek letters in red, German in green, and script in blue. The first paragraph must be capable of being used separately as a synopsis of the entire paper. In particular it should contain no bibliographic references. Please propose a heading for the odd numbered pages of less than 35 characters. Manuscripts, in triplicate, may be sent to any one of the editors. Please classify according to the scheme of Math. Reviews, Index to Vol. 39. Supply name and address of author to whom proofs should be sent. All other communications should be addressed to the managing editor, or Elaine Barth, University of California, Los Angeles, California 90024.

There are page-charges associated with articles appearing in the Pacific Journal of Mathematics. These charges are expected to be paid by the author's University, Government Agency or Company. If the author or authors do not have access to such Institutional support these charges are waived. Single authors will receive $\mathbf{5 0}$ free reprints; joint authors will receive a total of $\mathbf{1 0 0}$ free reprints. Additional copies may be obtained at cost in multiples of 50 .

The Pacific Journal of Mathematics is issued monthly as of January 1966. Regular subscription rate: $\$ 190.00$ a year (5 Vols., 10 issues). Special rate: $\$ 95.00$ a year to individual members of supporting institutions.

Subscriptions, orders for numbers issued in the last three calendar years, and changes of address should be sent to Pacific Journal of Mathematics, P.O. Box 969, Carmel Valley, CA 93924, U.S.A. Old back numbers obtainable from Kraus Periodicals Co., Route 100, Millwood, NY 10546.

The Pacific Journal of Mathematics at P.O. Box 969, Carmel Valley, CA 93924 (ISSN 0030-8730) publishes 5 volumes per year. Application to mail at Second-class postage rates is pending at Carmel Valley, California, and additional mailing offices. Postmaster: send address changes to Pacific Journal of Mathematics, P.O. Box 969, Carmel Valley, CA 93924.

PUBLISHED BY PACIFIC JOURNAL OF MATHEMATICS, A NON-PROFIT CORPORATION Copyright (C) 1988 by Pacific Journal of Mathematics 


\section{Pacific Journal of Mathematics}

\section{Vol. 131, No. 2 December, 1988}

Selman Akbulut and Henry Churchill King, Polynomial equations of immersed surfaces .................................... 209

Alberto Baider and Richard C. Churchill, The Campbell-Hausdorff group and a polar decomposition of graded algebra automorphisms ........2 219

Wayne C. Bell and John William Hagood, Separation properties and exact

Radon-Nikodým derivatives for bounded finitely additive measures . . . 237

Dennis J. Garity, James P. Henderson and David G. Wright, Menger

spaces and inverse limits ...............................249

B. Brent Gordon, Algebraically defined subspaces in the cohomology of a

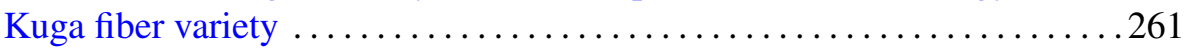

Jeffrey A. Hogan, Weighted norm inequalities for the Fourier transform on connected locally compact groups ........................... 277

Guojun Liao, A study of regularity problem of harmonic maps ..........291

Chin-pi Lu, Modules satisfying ACC on a certain type of colons ......... 303

Kunio Murasugi, Jones polynomials of periodic links

Hans Schoutens, Approximation properties for some non-Noetherian local

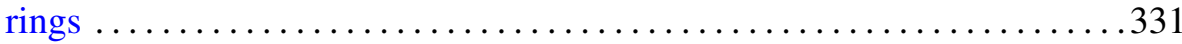

Peter Sjögren, Convergence for the square root of the Poisson kernel ...... 361 Alexandru Ion Suciu, The oriented homotopy type of spun 3-manifolds .393 